\title{
SOME PARTIAL DIFFERENTIAL EQUATIONS IN CLIFFORD ANALYSIS
}

\author{
ELENA OBOLASHVILI \\ Institute of Mathematics, Georgian Academy of Sciences \\ Tbilisi, Georgia \\ E-mail: helen@imath.kheta.ge
}

\begin{abstract}
Using Clifford analysis in a multidimensional space some elliptic, hyperbolic and parabolic systems of partial differential equations are constructed, which are related to the well-known classical equations. To obtain parabolic systems Clifford algebra is modified and some corresponding differential operator is constructed. For systems obtained the boundary and initial value problems are solved.
\end{abstract}

Introduction. The Clifford analysis has suggested us an idea to construct in a multidimensional space some partial differential equations which are related with the wellknown classical equations. Using Clifford algebra the operator generalizing the classical Cauchy-Riemann operator is considered in multidimensional space in [3], [7]. Applying this operator to the element of usual Clifford algebra one can get elliptic systems, and by applying to the element of some universal Clifford algebra, hyperbolic systems are obtained. The natural question has arised how to obtain parabolic systems. For this, we need to consider some modification of Clifford algebra. Thus, in a multidimensional space below elliptic, hyperbolic and parabolic systems are obtained, which are related with Laplace, wave and heat equations, respectively. An information about Clifford algebra one can find, for example, in [3], [5], [6], [7].

1. Some basic notions and definitions. Let $e_{1}, e_{2}, \ldots, e_{n}$ be an orthonormal base of the $n$-dimensional real vector space $R^{n}$ with respect to the usual scalar product. The universal Clifford algebra $R_{(n, s)}$ over $R^{n+1}$ has the basis

$$
e_{0}, e_{1}, \ldots, e_{n}, e_{1} e_{2}, \ldots, e_{n-1} e_{n}, \ldots, e_{1} e_{2} \ldots e_{n},
$$

by defining the basic multiplication rules as

$$
e_{0}^{2}=1, \quad e_{j}^{2}=-1, \quad j=1,2, \ldots, s,
$$

1991 Mathematics Subject Classification: Primary 46C20, Secondary 32G81.

The paper is in final form and no version of it will be published elsewhere. 


$$
e_{j}^{2}=1, \quad j=s+1, \ldots, n ; \quad e_{j} e_{k}+e_{k} e_{j}=0, \quad 1 \leq j<k \leq n
$$

where $e_{0}$ is its identity element. It is a real $2^{n}$-dimensional non-commutative $(n \geq 2)$ vector space. $R_{(n, n)} \equiv R_{(n)}$ is usual Clifford algebra. Thus, the basis consists of the elements $e_{A}=e_{\alpha_{1}} e_{\alpha_{2}} \ldots e_{\alpha_{k}}$, where $A:\left\{\alpha_{1}, \alpha_{2}, \ldots, \alpha_{k}\right\} \in\{1,2, \ldots, n\}$ and $1 \leq \alpha_{1}<$ $\alpha_{2}<\ldots<\alpha_{k} \leq n$. An arbitrary element $u \in R_{(n, s)}$ may be written as

$$
u=\sum_{A} u_{A} e_{A}, \quad u_{A} \in R, \quad 0 \leq \alpha_{1}<\alpha_{2}<\ldots<\alpha_{k} \leq n .
$$

For any $u, v \in R_{(n, s)}$ the product is defined as

$$
u \cdot v=\sum_{A, B} u_{A} v_{B} e_{A} e_{B}
$$

A convolution $u \longrightarrow \bar{u}$ called conjugation is defined by requiring that

$$
\bar{u}=\sum_{A} u_{A} \bar{e}_{A}
$$

with

$$
\bar{e}_{0}=e_{0}, \quad \bar{e}_{j}=-e_{j}, \quad j=1,2, \ldots, n, \quad \bar{e}_{A}=\bar{e}_{\alpha_{k}} \ldots \bar{e}_{\alpha_{1}} .
$$

Let a domain $\Omega \subset R^{n+1}$ and a function $u(x)$ :

$$
\Omega \longrightarrow R_{(n, s)} ; x\left(x_{0}, x_{1}, \ldots, x_{n}\right) \in \Omega .
$$

Consider the operators

$$
\bar{\partial}=\sum_{j=0}^{n} \frac{\partial}{\partial x_{j}} e_{j} \quad \partial=\frac{\partial}{\partial x_{0}} e_{0}-\sum_{j=1}^{n} \frac{\partial}{\partial x_{j}} e_{j} .
$$

Using (1), one can obtain the Coulomb operator

$$
\bar{\partial} \partial=\partial \bar{\partial}=\left[\sum_{j=0}^{s} \frac{\partial^{2}}{\partial x_{j}^{2}}-\sum_{j=s+1}^{n} \frac{\partial^{2}}{\partial x_{j}^{2}}\right] e_{0} .
$$

A function $u(x) \in C^{1}(\Omega)$ with values in $R_{(n, s)}$ is said to be regular in $\Omega$ if

$$
\bar{\partial} u=0, \quad u(x)=\sum_{A} u_{A}(x) e_{A} .
$$

For the regular function $u(x)$ with values in $R_{(n)}$ by virtue of $(7)$ we have:

$$
\Delta u=0,
$$

where the Laplace operator $\Delta$ is taken with respect to all $x_{0}, x_{1}, \ldots, x_{n}$. For the regular function $u(x)$ with values in $R_{(n, n-1)}$ by virtue of $(7)$ one can get the wave equation

$$
\Delta u-\frac{\partial^{2} u}{\partial x_{n}^{2}}=0
$$

where $\Delta$ is now taken with respect to variables $x_{0}, x_{1}, \ldots, x_{n-1}$.

2. Modified Clifford algebra and heat equation. Now in place of $\bar{\partial}$ we need to consider an operator which is connected with the heat equation. For this we consider 
some modified Clifford algebra (like in Grassman algebra). Let the multiplication rules be defined by

$$
\begin{gathered}
e_{o}^{2}=1, \quad e_{j}^{2}=-1, \quad j=1,2, \ldots, n-1 ; \quad e_{n}^{2}=0 \\
e_{j} e_{k}+e_{k} e_{j}=0, \quad j \neq k=1,2, \ldots, n .
\end{gathered}
$$

instead of (1) and equalities (4), (5), (6) remained unchanged. This algebra is denoted by $R_{(n)}^{0}$. Instead of (8) the following equation will be considered

$$
\left[\bar{\partial}-P_{(n)}\right] u(x)=0
$$

where a linear operator $P_{(n)} u$ is defined by the condition

$$
\partial P_{(n)} u=\frac{\partial u}{\partial x_{n}} \text {. }
$$

Using (6), (11) we have:

$$
\begin{gathered}
P_{(n)} u=-\sum_{A}(-1)^{k} u_{A n}(x) e_{A} \\
A_{\left(\alpha_{1}, \alpha_{2}, \ldots, \alpha_{k}\right)} \in\{0,1, \ldots, n-1\} .
\end{gathered}
$$

Now, it is obvious that if $u$ is the solution of (12), then it also is the solution of the heat equation:

$$
\Delta u=\frac{\partial u}{\partial x_{n}},
$$

where $\Delta$ is taken with respect to $x_{0}, x_{1}, \ldots, x_{n-1}$.

\section{Some partial cases.}

a) Let $u(x)$ have a vectorial form:

$$
u(x)=u_{0}(x) e_{0}-\sum_{j=1}^{n} u_{j}(x) e_{j}
$$

As is well known, if $u(x) \in R_{(n)}$, then (8) is equivalent to the Riesz system

$$
\begin{gathered}
\sum_{j=0}^{n} \frac{\partial u_{j}}{\partial x_{j}}=0, \\
\frac{\partial u_{j}}{\partial x_{k}}-\frac{\partial u_{k}}{\partial x_{j}}=0, \quad j, k=0,1, \ldots, n,
\end{gathered}
$$

which is for $n>1$ an overdetermined elliptic system.

Now, let $u(x) \in R_{(n, n-1)}$, then (8) is equivalent to the hyperbolic system

$$
\begin{gathered}
\sum_{j=0}^{n-1} \frac{\partial u_{j}}{\partial x_{j}}-\frac{\partial u_{n}}{\partial x_{n}}=0, \\
\frac{\partial u_{j}}{\partial x_{k}}-\frac{\partial u_{k}}{\partial x_{j}}=0, \quad j, k=0,1, \ldots, n .
\end{gathered}
$$

But if $u(x) \in R_{(n)}^{0}$ the solution of (12), where

$$
P_{(n)} u(x)=u_{n}(x) e_{o},
$$


then (12) is equivalent to the parabolic system

$$
\begin{gathered}
\sum_{j=0}^{n-1} \frac{\partial u_{j}}{\partial x_{j}}-u_{n}=0, \\
\frac{\partial u_{j}}{\partial x_{k}}-\frac{\partial u_{k}}{\partial x_{j}}=0, \quad j, k=0,1, \ldots, n .
\end{gathered}
$$

Thus, (18) and (19) can be considered as hyperbolic and parabolic analogues of Riesz system, respectively. If the scalar function $v(x)$ is any solution of $(9),(10),(15)$, then

$$
u_{j}=\frac{\partial v}{\partial x_{j}}, \quad j=0,1, \ldots, n
$$

is the solution of (17), (18), (19), respectively.

b) Let $n=2$ (the quaternionic case), and

$$
u(x)=u_{0}(x) e_{0}-u_{1}(x) e_{1}-u_{2}(x) e_{2}-u_{12}(x) e_{1} e_{2} .
$$

Let $u(x) \in R_{(2)}$, then, as is known, (8) is equivalent to the Moisil-Theodorescu system [9], which we have written in a vectorial form [10a]:

$$
\begin{gathered}
\operatorname{div} U=0, \\
\operatorname{grad} \varphi+\operatorname{rot} U=0
\end{gathered}
$$

where $U\left(u_{0}, u_{1}, u_{2}\right)$ - three-component vector, $\varphi \equiv u_{12}$ - scalar function, operations div, grad, rot are taken with respect to $x_{0}, x_{1}, x_{2}$. Let $u(x) \in R_{(2,1)}$. Note that in the cases $R_{(2,1)}$ and $R_{(2,0)}$ equation (8) gives us the hyperbolic system of the same form. That is why it is sufficient to consider only one of them. Thus, (8) is equivalent to the system:

$$
\begin{aligned}
\frac{\partial u_{0}}{\partial x_{0}}+\frac{\partial u_{1}}{\partial x_{1}}-\frac{\partial u_{2}}{\partial x_{2}}=0, & \frac{\partial u_{12}}{\partial x_{0}}+\frac{\partial u_{2}}{\partial x_{1}}-\frac{\partial u_{1}}{\partial x_{2}}=0 \\
\frac{\partial u_{0}}{\partial x_{1}}-\frac{\partial u_{1}}{\partial x_{0}}+\frac{\partial u_{12}}{\partial x_{2}}=0, & \frac{\partial u_{12}}{\partial x_{1}}-\frac{\partial u_{2}}{\partial x_{0}}+\frac{\partial u_{0}}{\partial x_{2}}=0 .
\end{aligned}
$$

Considering the complex functions

$$
\begin{aligned}
& \varphi(x)=u_{0}+u_{12}-i\left(u_{1}+u_{2}\right), \\
& \psi(x)=u_{0}-u_{12}-i\left(u_{1}-u_{2}\right),
\end{aligned}
$$

(22) can be written as

$$
\begin{gathered}
2 \frac{\partial \varphi}{\partial \bar{z}}+i \frac{\partial \bar{\varphi}}{\partial x_{2}}=0, \quad 2 \frac{\partial \psi}{\partial \bar{z}}-i \frac{\partial \bar{\psi}}{\partial x_{2}}=0, \quad z=x_{0}+i x_{1} \\
2 \frac{\partial}{\partial \bar{z}}=\frac{\partial}{\partial x_{0}}+i \frac{\partial}{\partial x_{1}} .
\end{gathered}
$$

These equations are the partial case of the metaparabolic equations whose general form was considered in [1]. Some initial value problems of such equations were considered in [10]. 
Let now $u(x) \in R_{(2)}^{0}$, then since $P_{(2)} u=u_{2} e_{0}-u_{12} e_{1}$, (12) is equivalent to the parabolic system

$$
\begin{array}{cl}
\frac{\partial u_{0}}{\partial x_{0}}+\frac{\partial u_{1}}{\partial x_{1}}-u_{2}=0 & \frac{\partial u_{12}}{\partial x_{0}}+\frac{\partial u_{2}}{\partial x_{1}}-\frac{\partial u_{1}}{\partial x_{2}}=0 \\
\frac{\partial u_{0}}{\partial x_{1}}-\frac{\partial u_{1}}{\partial x_{0}}+u_{12}=0 & \frac{\partial u_{12}}{\partial x_{1}}-\frac{\partial u_{2}}{\partial x_{0}}+\frac{\partial u_{0}}{\partial x_{2}}=0 .
\end{array}
$$

Considering complex functions

$$
w_{1}=u_{0}-i u_{1}, \quad w_{2}=u_{12}-i u_{2},
$$

(25) can be written in the complex form

$$
2 \frac{\partial w_{1}}{\partial \bar{z}}+i \bar{w}_{2}=0, \quad 2 \frac{\partial w_{2}}{\partial \bar{z}}+i \frac{\partial \bar{w}_{1}}{\partial x_{2}}=0 .
$$

c) Let now $n=3$ and

$$
u(x)=u_{0} e_{0}-\sum_{j=1}^{3} u_{j} e_{j}-\sum_{1=j<k}^{3} u_{j k} e_{j} e_{k}-u_{123} e_{1} e_{2} e_{3} .
$$

If $u(x) \in R_{(3,1)}$, the equation (8) is equivalent to the hyperbolic system:

$$
\begin{array}{r}
\operatorname{div} U-\frac{\partial \varphi}{\partial x_{3}}=0, \quad \operatorname{div} V+\frac{\partial \psi}{\partial x_{3}}=0, \\
\operatorname{grad} \psi+\operatorname{rot} U+\frac{\partial V}{\partial x_{3}}=0, \quad \operatorname{grad} \varphi+\operatorname{rot} V-\frac{\partial U}{\partial x_{3}}=0,
\end{array}
$$

where $U \equiv\left(u_{0}, u_{1}, u_{2}\right), V \equiv\left(u_{123}, u_{23},-u_{13}\right)$ are three-component vectors, $u_{12} \equiv \psi$, $u_{3} \equiv \varphi$ - scalars, the operators grad, div, rot are taken with respect to $x_{0}, x_{1}, x_{2}$. Note, that if $\varphi=\psi \equiv 0$ and $x_{3} \equiv t$ is a time variable, then (28) are Maxwell's equations (in a vacuum). But, if the unknown quantities in (28) do not depend on $x_{3}$, this system forms two seperated Moisil-Theodorescu systems (21).

Let $u(x) \in R_{(3)}^{(0)}$, then by using (27), equation (12) is equivalent to the parabolic system having that

$$
\begin{gathered}
P_{(3)} u=\varphi e_{0}-u_{13} e_{1}-u_{23} e_{2}+u_{123} e_{1} e_{2}, \\
\operatorname{div} U-\varphi=0, \quad \operatorname{div} V+\frac{\partial \psi}{\partial x_{3}}=0, \\
\operatorname{grad} \psi+\operatorname{rot} U+V=0, \quad \operatorname{grad} \varphi+\operatorname{rot} V-\frac{\partial U}{\partial x_{3}}=0 .
\end{gathered}
$$

Let the scalar functions $f_{1}, f_{2}$ be solutions of (10) or (15) for $n=3$. Then

$$
U=\operatorname{grad} f_{1}, \quad V=\operatorname{grad} f_{2}, \quad \varphi=\frac{\partial f_{1}}{\partial x_{3}}, \quad \psi=-\frac{\partial f_{2}}{\partial x_{3}}
$$

are the solutions of (28), (29) respectively.

4.Boundary and initial value problems. Let $S_{(n)}$ be the half hyperspace $x_{n} \geq$ $0(n \geq 1)$. The following problems are correctly posed: 
a) Find a bounded solution of (17) or (19) in $S_{(n)}$, when only one condition is given on the boundary:

$$
u_{j}=f\left(x_{0}, \ldots, x_{n-1}\right), \quad \text { for } x_{n}=0,
$$

where $j$ is fixed and takes one of the values $0,1, \ldots, n$.

b) Find a bounded solution of (18) in $S_{(n)}$, when only two conditions are given on the boundary: for $x_{n}=0 u_{j}, u_{n}$ are given, where $j$ is a fixed number from $\{0,1, \ldots, n-1\}$; or $u_{j}, \frac{\partial u_{j}}{\partial x_{n}}$ are given where $j \in\{0,1, \ldots, n\}$ is fixed. Note that the number of given conditions on $x_{n}=0$ does not depend on $n$.

Let $\Phi(U, \psi)$ and $\Psi(V, \varphi)$ be four-component vectors, constructed by the solutions of (28) or $(29)$.

c) Find a bounded solution of (28) in $S_{(3)}$, when both vectors: $\Phi$ and $\Psi$ for $x_{3}=0$ are given.

d) Find a bounded solution of (29) in $S_{(2)}$, when any four quantities from eight unknowns for $x_{3}=0$ are given.

Analogous problems can be considered in $S_{(12)}$ for equations $(21),(22),(25)$. The unique solutions of all these problems in certain classes can be ropresented in quadratures using, for example, Fourier integral transform.

Now I want to note the following: The generalized Moisil-Theodorescu system

$$
\begin{gathered}
\operatorname{div} U+(A \cdot U)=0 \\
\operatorname{grad} \varphi+\operatorname{rot} U+[B \times U]+C \varphi=0,
\end{gathered}
$$

where $A, B, C$ are given three component vectors; div, grad and rot are taken with respect to $x_{0}, x_{1}, x_{2}$, was first considered in [10a] in 1975 (Russian), then it was also considered in [10b] (English). To define the solution of (30) in $S_{(2)}$ it is sufficient to give on $x_{2}=0$ two boundary conditions, but in the bounded domain it is not sufficient [2]. Let $S$ be a domain bounded by the closed smooth surface $\Gamma$ and $L$ be a closed smooth line on $\Gamma$, such that its orthogonal projection $L_{0}$ on the plane $x_{2}=0$ bounds the domain of variables $x_{0}, x_{1}$ for $S$. The following problem was posed and solved in [10a]:

Find a regular solution of (30) in $S$ by the conditions:

$$
\begin{gathered}
u_{0}(x)=f_{0}(x), \quad \varphi(x)=f(x), \quad x \in \Gamma, \\
\alpha u_{1}(x)+\beta u_{2}(x)=g(x), \quad x \in L,
\end{gathered}
$$

where $f_{0}, f$ and $\alpha, \beta, g$ are given functions on $\Gamma$ and $L$ respectively.

Recently I have seen the article [8], where the system (30) is considered and some of our old results are obtained again. Unfortunately, the author, Huang Liede, perhaps, does not know our papers [10a], [10b]. Moreover, for nonhomogeneous Moisil-Theodorescu system the boundary conditions of type (31) are considered in [4], and there is no reference to my papers.

Acknowledgements. I should like to express my great thanks to professor J. Ławrynowicz for the invitation to take part in symposium and giving me an opportunity to visit the remarkable Banach International Mathematical Center, where mathematicians of all world can meet and discuss new problems. Then I express my appreciation to professors 
B. Bojarski and J. Ławrynowicz, Institute of Mathematics of the Polish Academy of Sciences for financial support and the hospitality offered me during my stay in Poland.

\section{References}

[1] M. Begher, R. Gilbert, Piecewise continuous solution of pseudoparabolic equations in two space dimensions, Proc. Royal Soc. Edinburgh 81A (1978), 153-173.

[2] A. Bitsadze, Boundary value problems of elliptic equations of second order, Nauka, Moscow, 1966 (Russian).

[3] F. Brack, R. Delanghe, F. Sommen, Clifford Analysis, Pitman, London, 1982.

[4] A. Dzhuraev, On the Moisil-Theodorescu system, P.D.E. with complex analysis, (editors H. Begher and A. Jeffrey), Longman Scient. and Techn. 1992, 186-203.

[5] K. Gurlebeck, W. Sproßig, Quaternionic analysis and elliptic boundary value problems, Akademie-Verlag, Berlin 1989.

[6] K. Habetha, Function theory in algebras. Complex analysis, Methods, Trends and Applications. Ak. Verlag, Berlin 1983, 225-237.

[7] V. Iftime, Fonctions hypercomplexes. Bull. Math. R. S. de Roumanie 9(57) (1965), 279-332.

[8] H. Liede, The existence and uniqueness theorems of the linear and nonlinear R.-H. problems for the generalized holomorphic vector of the second kind, Acta Math. Sci. Engl. Ed. 10 no. 2 (1990), 185-199.

[9] G. Moisil, N. Theodorescu, Fonctions holomorphes dans l'espace, Mathematica 5 (1931).

[10a] E. Obolashvili, Space generalized holomorphic vectors, Diff. Urav. T.XI.1, 1975, 108-115. Minsk (Russian).

[10b] E. Obolashvili, Effective solutions of some boundary value problems in two and three dimensional cases, Functional analytic methods in complex analysis and applications to PDE, 1988.Trieste, 149-172.

[10c] E. Obolashvili, Some boundary value problems for metaparabolic equations (Russian). Proceeding of I. Vekua Inst. of Applied math. T.1, N.1, 1985, 161-164. 